\title{
Anisotropy of Particle Fluxes in Primary Cosmic Rays Measured with the Alpha Magnetic Spectrometer on the ISS
}

\author{
Iris Gebauer*广 \\ Karlsruhe Institute of Technology, Institute for Experimental Particle Physics, D-76131, \\ Karlsruhe, Germany \\ E-mail: gebauerekit.edu \\ M. Graziani \\ Karlsruhe Institute of Technology, Institute for Experimental Particle Physics, D-76131 \\ Karlsruhe, Germany
}

\section{J. Casaus, M. Molero, C. Mana, M. A. Velasco}

Centro de Investigaciones Energéticas, Medioambientales y Tecnológicas (CIEMAT), E-28040

Madrid, Spain

\section{Gervasi, G. La Vacca, P.G. Rancoita}

INFN sez. Milano-Bicocca, Piazza della Scienza, 3 - 20126 Milano, Italy

Physics Department, University of Milano-Bicocca, Piazza della Scienza, 3 - 20126 Milano,

Italy

\begin{abstract}
An analysis of anisotropies in the arrival directions of galactic electrons and positrons, as well as protons, Helium, Carbon and Oxygen has been performed with the Alpha Magnetic Spectrometer on the International Space Station using the first 6.5 years (electrons and positrons) and the first 7.5 years (protons, Helium, Carbon, Oxygen) of data taking. Results on a dipole signal in Galactic coordinates are reported.

The arrival directions of all studied cosmic ray species are consistent with isotropy. For energies above $16 \mathrm{GeV}$ a limit of $\delta<0.019(\delta<0.005)$ at the $95 \%$ confidence level is obtained for positrons (electrons). For rigidities above $200 \mathrm{GV}$ a limit of $\delta<0.0038(\delta<0.0036, \delta<0.019$, $\delta<0.017$ ) at the $95 \%$ confidence level is obtained for protons (Helium, Carbon, Oxygen). The quoted limits are based on a total of $9.9 \times 10^{4}\left(1.3 \times 10^{6}, 2 \times 10^{6}, 2.2 \times 10^{6}, 6.1 \times 10^{4}, 6.3 \times 10^{4}\right)$ positron (electron, proton, Helium, Carbon, Oxygen) events.
\end{abstract}

36th International Cosmic Ray Conference -ICRC2019-

July 24th - August 1st, 2019

Madison, WI, U.S.A.

\footnotetext{
* Speaker.

${ }^{\dagger}$ on Behalf of the AMS Collaboration
} 


\section{Introduction}

The precise measurements of Cosmic Ray (CR) fluxes performed by the Alpha Magnetic Spectrometer (AMS-02) onboard the ISS, have revealed multiple unexpected features which cannot be explained in the standard paradigm of cosmic ray transport: for example, the spectra of primary cosmic rays like protons, Helium, Carbon and Oxygen $[1,2,3]$ and secondary cosmic rays like Lithium, Berylium and Boron [4] as well as mixed species like Nitrogen [5] exhibit a hardening at rigidities of above $200 \mathrm{GV}$, which might be caused by the presence of a local CR accelerator dominating the local CR spectra above a few hundred GV. Further, the positron fraction shows a rise above a few $\mathrm{GeV}$ with a slope decreasing logarithmically with energy above $30 \mathrm{GeV}$ and above $200 \mathrm{GeV}$ the positron fraction is no longer increasing with energy [6]. This behaviour originates from the electron and positron fluxes dependence on energy [7, 8, 9]. Above around $20 \mathrm{GeV}$ and up to $200 \mathrm{GeV}$ the electron flux decreases more rapidly with energy than the positron flux, that is, the electron flux is softer than the positron flux [9]. Above $25 \mathrm{GeV}$ the positron flux shows a significant excess compared to the low-energy power-law trend and above $284 \mathrm{GeV}$ the positron flux shows a sharp dropoff [8]. The fluxes of electrons and positrons indicate the presence of a new source of energetic positrons, which dominates above the background of positrons produced in CR collisions at high energies.

These features cannot be explained within our current understanding of galactic cosmic ray transport. They may be related to the presence of unaccounted astrophysical sources, e.g. local supernova remnants or Wolf-Rayet stars might be the source of the hard proton and helium component, which dominates the respective CR spectra above a few hundreds of GV, pulsars might produce energetic electron-positron pairs in their strong rotating magnetic fields, which are able to explain the observed rise and flattening on the positron fraction.

If the observed features are indeed caused by a limited number of astrophysical point sources, such a point source needs to be relatively close by. Otherwise fast energy losses for electrons and positrons or escape from the galaxy for cosmic ray nuclei would significantly soften the CR spectra. A limited number of close-by point sources could induce some degree of anisotropy in the $\mathrm{CR}$ arrival directions. Therefore it is worth to measure and characterize the angular distribution of $\mathrm{CR}$ arrival directions. Anisotropy searches therefore provide complementary information to constrain the origin of the observed features and the propagation and production mechansims of CRs in the Galaxy.

In this study we analized a sample of electrons and positrons taken in the first 6.5 years of AMS-02 data taking between $16 \mathrm{GeV}$ and $350 \mathrm{GeV}$ and a sample of protons, Helium, Carbon and Oxygen nuclei above $18 \mathrm{GV}$ taken in the first 7.5 years of AMS data taking. A search for absolute 3-dimensional dipole anisotropies in the arrival directions of these CR species was performed.

\section{The AMS-02 Detector}

AMS-02 is a large acceptance multipurpose particle detector, designed to measure the fluxes of individual cosmic ray species at $\mathrm{GeV}$ and $\mathrm{TeV}$ energies. It was installed on 19 May 2011 onboard the International Space Station (ISS) and has been continuously taking data since then, collecting more than 140 billion events of galactic CRs. The detector will remain on the ISS and 
continue to take data until the end of ISS operation which is currently planned for 2024. The detector consists of nine precision silicon tracker planes with two outer planes, 1 and 9 , and the inner tracker, planes 2 to 8 ; a transition radiation detector (TRD); four planes of time of flight (TOF) counters; a permanent magnet; an array of anticoincidence counters (ACC), inside the magnet bore; a ring imaging Čerenkov detector (RICH); and an electromagnetic calorimeter (ECAL). A detailed dectector description can also be found in $[1,2,3,4,5,6,7,8,9,10,11]$.

\section{Data Selection}

Anisotropy searches rely on directional information for single events. To preserve single particle information a cut based selection is performed.

For electrons and positrons the basic quality selection follows the description in $[6,7,8,9]$, but no template fits in ECAL and TRD estimators are performed to identify particles. The reduction of the proton background is achieved by means of the TRD, the ECAL and the tracker. Events are selected by requiring a minimum of 8 TRD hits consistent with the tracker track, a cluster of hits in the ECAL, and a measured velocity $\beta \sim 1$ in the TOF consistent with a downward-going $\mathrm{Z}=1$ particle. Protons are rejected by requiring a good energy-momentum matching and explicit cuts on the ECAL and TRD estimators. The overall selection efficiency for positrons and electrons is reduced compared to the template fit analysis in $[7,6,8,9]$. The remaining sample contains $9.9 \times 10^{4}$ positrons and $1.3 \times 10^{6}$ electrons above $16 \mathrm{GeV}$ with negligible proton contamination. The selected events are grouped into 5 cumulative energy ranges from 16 to $350 \mathrm{GeV}$ according to their measured energy in the ECAL. The minimum energies are 16, 25, 40, 65 and $100 \mathrm{GeV}$, respectively.

The proton, Helium, Carbon and Oxygen selection follows the one described in [1, 2, 3]. The first step of the selection requires the preselection of events in which the velocity was measured by four TOF layers being consistent with down-going particles. Further cuts are made requiring the charge consistency with the respective species and requiring the track, reconstructed by the 7 inner layers, to pass through the outer layers 1 and 9 for protons and to pass through the outer layer 1 for Helium, Carbon and Oxygen to satisfy additional track fitting quality criteria. The selected events are grouped into 9 cumulative rigidity bins with minimum rigidites 18, 30, 45, 80, 150, 200, 300, 500 and $1000 \mathrm{GV}$. In addition, to select only primary CRs, well above the geomagnetic cutoff, the measured rigidity is required to be greater than 1.2 times the maximum geomagnetic cutoff within the AMS field of view.

\section{Method}

The ISS orbit inclination of $51.6^{\circ}$ relative to the Earth's equator leads to a non-uniform sky coverage of the AMS-02 detector. The left side of Fig. 1 shows the AMS-02 pointing direction over a period of 2.5 years in galactic coordinates. Regions with high exposure around the Earth's north and south pole are clearly visible. The finite detector acceptance of about $35^{\circ}$ for a proton selection and $25^{\circ}$ for an electron selection, smears out the pointing direction, leading to rings of high exposure around the Earth's north and south poles. The right side of Fig. 1 shows the proton 
sky above $40 \mathrm{GV}$ as seen by AMS-02. The nearly full sky coverage allows to search for a full 3-dimensional dipole in CR arrival directions.

The CR arrival directions are binned in sky maps in galactic coordinates $(l, b)$ containing the number of observed particles using the HEALPix ${ }^{1}$ pixelization, which guarantees a regular sampling of the CR angular distribution.
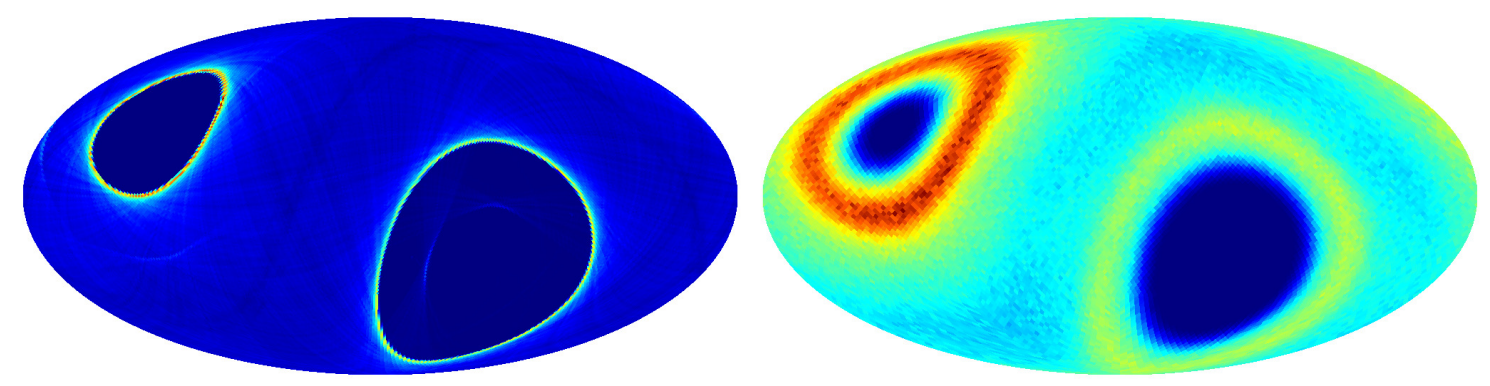

Figure 1: Left: The AMS-02 pointing direction over a period of 2.5 years. The color coding illustrates the number of seconds the z-axis of the detector spends pointing to a certain position in the sky. Right: The proton sky above $40 \mathrm{GeV}$ as observed by AMS-02.

The efficiency with wich particles can be detected by AMS-02 depends on the trigger rate which changes with geomagnetic latitude as a result of the bending of the Earth's magnetic field lines. Towards the poles a higher rate of low rigidity particles is able to reach ISS orbit, while at the equator these particles are reflected by the Earth's magnetic field. In addition, single cut efficiencies may have a dependence on detector position.

Above $16 \mathrm{GeV}$ all $\mathrm{CR}$ species share the same exposure ${ }^{2}$, while selection efficiencies and acceptance may be different as a function of detector position.

The search for anisotropies makes it necessary to first estimate an accurate representation of how an isotropic sky would look like for any given particle selection. For this so-called reference maps are used. A reference map is any map providing our best guess of what an isotropic sky would look like for the respective CR species, collected by the same detector in the same datataking period. Any deviation between the reference map and the data might be detected as a signal. Here we present results for absolute anisotropies which are derived from comparing the data to socalled IsoSky maps simulated from data of the same CR species. Several other methods yielding relative anisotropies, e.g. the use of protons as a reference for positrons or the use of the same CR species at different energies have been applied. These methods are discussed in [12]. The method to create IsoSky maps employed in this analysis was first introduced in [13]. It projects the detector's field of view into the galactic sky and then weights the accumulated livetime in this field of view with the detector efficiencies for the respective particle selection according to the following algorithm: Start by getting a list of particle incoming directions $(\theta, \phi)$ in detector coordinates from the selected data events. Then, for every second of data taking:

1. Draw randomly $\mathbf{N}$ sets of incoming directions $(\theta, \phi)_{i}$ from this list. (Geometric acceptance is preserved.)

\footnotetext{
${ }^{1}$ http://healpix.sourceforge.net

${ }^{2}$ below $16 \mathrm{GeV}$ the East-West effect may induce differences in the exposure of particle of different charges
} 
2. Calculate the galactic arrival direction $\Psi_{i}$ from the drawn incoming directions and the detector position in the respective second. (The geometric acceptance is projected onto the galactic sky.)

3. Weight the events with the detector livetime $T_{\exp }$ in this second to account for busy trigger and a time and position dependent correction factor $\varepsilon(t, \vec{x})$, to account for time and position dependent efficiencies.

An example of a resulting reference map, called IsoSky map, for protons can be found in the top left of Fig. 2.

Using a likelihood fit proceedure, the normalized ratio of data map and reference map is expanded into spherical harmonics:

$$
\phi_{i}=\sum_{\ell=0}^{\ell_{\max }} \sum_{m=-\ell}^{\ell} a_{\ell m} Y_{\ell m}\left(l_{i}, b_{i}\right),
$$

where $\left(l_{i}, b_{i}\right)$ is the position of the $i^{t h}$ pixel in galactic coordinates. The dipole for $\ell=1$ is fully described by three orthogonal functions aligned in galactic coordinates with directions: $Y_{10}$ along the North-South (NS) direction perpendicular to the galactic plane, $Y_{11}$ along the Forward-Backward (FB) direction with respect to the galactic center and $Y_{1-1}$ along the East-West (EW) direction tangent to the orbit of the sun around the galactic center. It is then worth to study these three directions both separately and combined to determine the total omnidirectional dipole magnitude

$$
\delta=\sqrt{\rho_{N S}^{2}+\rho_{F B}^{2}+\rho_{E W}^{2}} .
$$

where the three dipole coefficients are defined as

$$
\rho_{N S}=\sqrt{\frac{3}{4 \pi}} a_{10}, \quad \rho_{F B}=\sqrt{\frac{3}{4 \pi}} a_{11}, \quad \rho_{E W}=\sqrt{\frac{3}{4 \pi}} a_{1-1} .
$$

This procedure proved to be stable against different map resolutions and sample statistics.

The method was applied to protons, Helium, Carbon, Oxygen, electrons and positrons. No significant deviation from isotropy was found for any CR species. Other reference maps, as described in [12] were used as a cross check, yielding compatible results.

\section{Anisotropy in the arrival direction of protons, Helium, Carbon and Oxygen}

Figure 2 shows the dipole components for proton arrival directions as function of the minimum rigidities. No significant deviation from isotropy can be observed. The corresponding 95\% C.L. upper limits on the dipole amplitude for different rigidities are reported on the left side of the top panel of Fig. 3. The limit obtained for the rigidity range above $200 \mathrm{GV}$ is $\delta<0.0038$. For rigidties above $70 \mathrm{GV}$ the measurement is limited by statistics, at low rigidities systematics on the efficiency corrections limit the sensitivity to $0.1 \%$.

Fig. 3 also shows the 95\% C.L. upper limits on the dipole amplitude for different rigidities for Helium, Carbon and Oxygen. The limit obtained at the $95 \%$ confidence level for the rigidity range above $200 \mathrm{GV}$ is $\delta<0.0036$ for Helium, $\delta<0.019$ for Carbon and $\delta<0.017$ for Oxygen. 

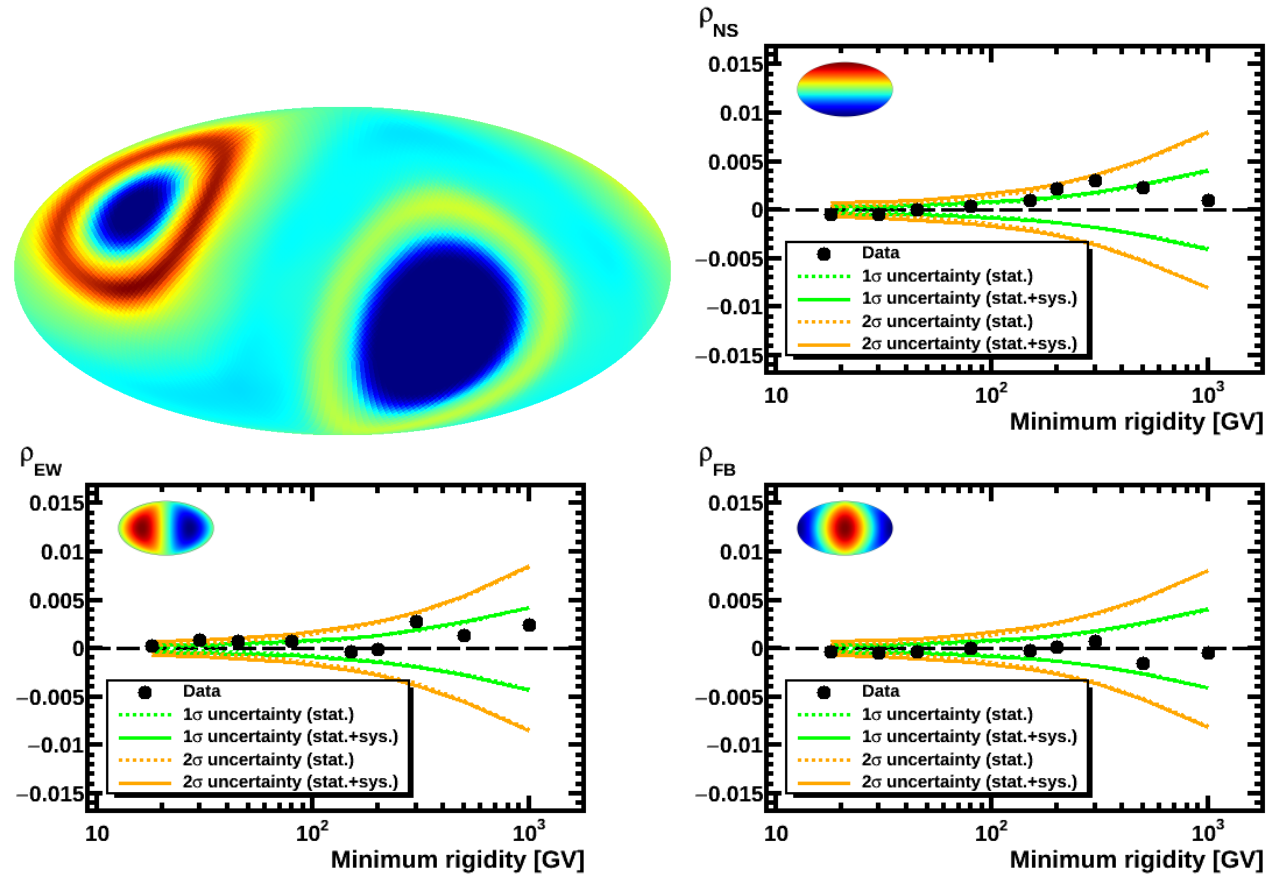

Figure 2: Top left: IsoSky map for protons above $40 \mathrm{GV}$ in galactic coordinates. Other figures: Amplitudes of the $\rho_{N S}$ (top right), $\rho_{E W}$ (bottom left) and $\rho_{F B}$ (bottom right) dipole components in the proton arrival directions obtained for different rigidity ranges. The black dots represent the fitted dipole components, the full green and orange lines represent the $1 \sigma$ and $2 \sigma$ statistical uncertainty. The efficiency corrections on the IsoSky map introduce systematics. The combined statistical and systematic uncertainties are shown as the green dashed and orange dashed line.

To check for possible unknown systematics in the creation of the IsoSky maps a similar analyses were performed using low energy proton, Helium, Carbon and Oxygen events as a reference for the respective high energy events, as described in [12]. The obtained results are consistent with the results reported here.

\section{Anisotropy in the arrival directions of positrons and electrons}

In Fig. 4 the 95\% C.L. upper limits on the dipole amplitude for positrons (left) and electrons (right) are shown as function of the minimum energy. No significant deviation from isotropy can be observed. The limit obtained for the energy range from 16 to $350 \mathrm{GeV}$ is $\delta<0.019$ for positrons and $\delta<0.005$ for electrons as reported in [8,9].

To check for possible unknown systematics in the creation of the IsoSky maps a similar analysis was performed using protons and electrons as a reference for positrons and protons as a reference for electrons. The obtained results are consistent with the results reported here.

\section{Conclusions}

A search for a dipole signal in the arrival directions of positrons, electrons, protons, Helium, Carbon and Oxygen was performed using 6.5 years of data (positrons and electrons) and 7.5 years 

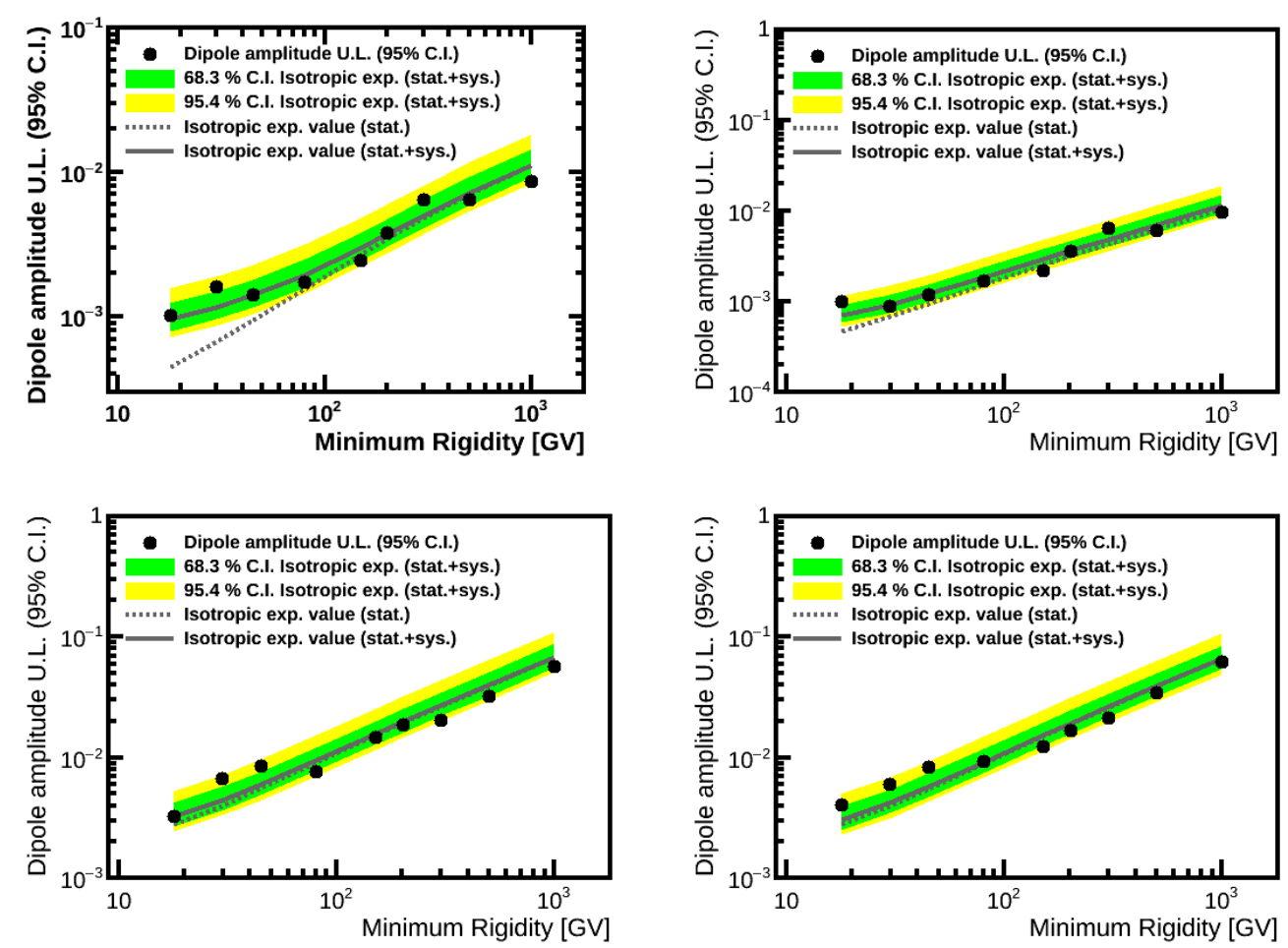

Figure 3: The 95\% C.L. upper limit for the omnidirectional dipole amplitude for protons (top left), Helium (top right), Carbon (bottom left) and Oxygen (bottom right). The upper limit is calculated from monte carlo using simulated dipoles and isotropic reference maps. A bayesian 95\% C.L. is computed from the distribution of true dipole strengths for the dipole strength measured on data. Also shown is the isotropic expectation calculated from a simulated isotropic signal. The gray dashed line indicates the isotropic expectation value based on only statistical uncertainties, the gray full line indicated the isotropic expectation value based on statistical and systematic uncertainty. The isotropic expectation is quoted as the 68.3\% C.L. (green) and 95.4\% C.L. (yellow) of the reconstructed $\delta$ distribution.
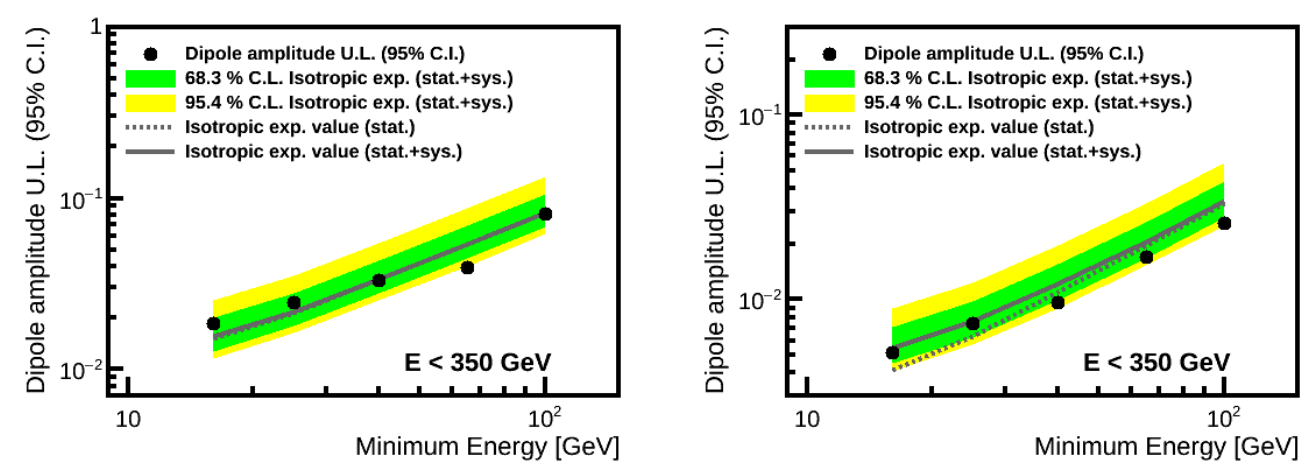

Figure 4: The 95\% C.L. upper limits on the omnidirectional dipole amplitude for positrons (left) and electrons (right) as function of the minimum energy. See caption of Fig. 3 for details.

of data (protons, Helium, Caron, Oxygen) taken with the AMS-02 detector onboard the ISS. No significant deviation from isotropy was observed. A 95\% confidence level upper limit of $\delta<0.019$ 
and $\delta<0.005$ was obtained for the energy range 16 to $350 \mathrm{GeV}$ for positrons and electrons, respectively. For protons (Helium, Carbon, Oxygen) a 95\% confidence level upper limit of $\delta<$ 0.0038 ( $\delta<0.0036, \delta<0.019, \delta<0.017$ ) was obtained for the rigidity range above $200 \mathrm{GV}$. Each analysis was performed using independent references maps. The obtained results are consistent.

\section{References}

[1] M. Aguilar et al., "Precision Measurement of the Proton Flux in Primary Cosmic Rays from Rigidity $1 \mathrm{GV}$ to $1.8 \mathrm{TV}$ with the Alpha Magnetic Spectrometer on the International Space Station," Phys. Rev. Lett., vol. 114, p. 171103, Apr 2015.

[2] M. Aguilar et al., "Precision Measurement of the Helium Flux in Primary Cosmic Rays of Rigidities 1.9 GV to 3 TV with the Alpha Magnetic Spectrometer on the International Space Station," Phys. Rev. Lett., vol. 115, p. 211101, Nov 2015.

[3] M. Aguilar et al., "Observation of the Identical Rigidity Dependence of $\mathrm{He}, \mathrm{C}$, and O Cosmic Rays at High Rigidities by the Alpha Magnetic Spectrometer on the International Space Station," Phys. Rev. Lett., vol. 119, no. 25, p. 251101, 2017.

[4] M. Aguilar et al., "Observation of New Properties of Secondary Cosmic Rays Lithium, Beryllium, and Boron by the Alpha Magnetic Spectrometer on the International Space Station," Phys. Rev. Lett., vol. 120, no. 2, p. 021101, 2018.

[5] M. Aguilar et al., "Precision Measurement of Cosmic-Ray Nitrogen and its Primary and Secondary Components with the Alpha Magnetic Spectrometer on the International Space Station," Phys. Rev. Lett., vol. 121, no. 5, p. 051103, 2018.

[6] L. Accardo et al., "High Statistics Measurement of the Positron Fraction in Primary Cosmic Rays of 0.5-500 GeV with the Alpha Magnetic Spectrometer on the International Space Station," Phys. Rev. Lett., vol. 113, p. 121101, Sep 2014.

[7] M. Aguilar et al., "Electron and Positron Fluxes in Primary Cosmic Rays Measured with the Alpha Magnetic Spectrometer on the International Space Station," Phys. Rev. Lett., vol. 113, p. 121102, Sep 2014.

[8] M. Aguilar et al., "Towards Understanding the Origin of Cosmic-Ray Positrons," Phys. Rev. Lett., vol. 122, no. 4, p. 041102, 2019.

[9] M. Aguilar et al., "Towards Understanding the Origin of Cosmic-Ray Electrons," Phys. Rev. Lett., vol. 122, no. 10, p. 101101, 2019.

[10] M. Aguilar et al., "First Result from the Alpha Magnetic Spectrometer on the International Space Station: Precision Measurement of the Positron Fraction in Primary Cosmic Rays of 0.5-350 GeV," Phys. Rev. Lett., vol. 110, p. 141102, Apr 2013.

[11] M. Aguilar et al., "Precision measurement of the $\left(e^{+}+e^{-}\right)$flux in primary cosmic rays from $0.5 \mathrm{gev}$ to 1 tev with the alpha magnetic spectrometer on the international space station," Phys. Rev. Lett., vol. 113, p. 221102, Nov 2014.

[12] I. Gebauer, K. F. Bindel, M. Graziani, S. Zeissler, J. Casaus, C. Mana, M. A. Velasco, M. Gervasi, G. La Vacca, and P. G. Rancointa, "Measurement of anisotropies in cosmic ray arrival directions with the Alpha Magnetic Spectrometer on the ISS," PoS, vol. ICRC2017, p. 186, 2018.

[13] I. Gebauer, "Direction and time dependent fluxes with AMS-02," PoS, vol. ICRC2015, p. 404, 2016. 